\title{
STRONGLY ZERO-DIMENSIONAL BISPACES
}

\author{
HANS-PETER A. KÜNZI
}

(Received 5 May 1990)

Communicated by J. H. Rubinstein

\begin{abstract}
Let $\mathscr{C}_{b}$ be the admissible functorial quasi-uniformity on the completely regular bispaces which is spanned by the upper quasi-uniformity on the real line. Answering a question posed by $B$. Banaschewski and G. C. L. Brümmer in the affirmative we show that $\mathscr{C}_{b} X$ is transitive for every strongly zero-dimensional bispace $X$.

1991 Mathematics subject classification (Amer. Math. Soc.): 54 E 15, 54 E 55, 18 B 30, 54 F 45. Keywords and phrases: strongly zero-dimensional, semi-continuous quasi-uniformity, functorial quasi-uniformity, completely regular bispace, transitive quasi-uniformity, non-archimedeanly quasi-pseudo-metrizable.
\end{abstract}

\section{Introduction}

Let us recall that a (quasi-)uniform space is said to be transitive if its filter of entourages has a base of transitive entourages. In [1] B. Banaschewski and G. C. L. Brümmer prove that each admissible functorial uniformity on the completely regular topological spaces is transitive exactly on the strongly zero-dimensional spaces [1, Theorem 1.3]. This contrasts with their result that among those functorial quasi-uniformities which are admissible on all completely regular bispaces (that is, all pairwise completely regular bitopological spaces) some are and others are not transitive on the strongly zerodimensional bispaces. For example the fine quasi-uniformity on a strongly zero-dimensional (completely regular) bispace may fail to be transitive [1, Proposition 1.6], but the coarsest functor $\mathscr{C}_{b}^{*}$ that puts admissible quasiuniformities on the completely regular bispaces is transitive precisely on the

(C) 1992 Australian Mathematical Society 0263-6115/92 \$A2.00+0.00 
strongly zero-dimensional bispaces [1, Proposition 1.4]. Banaschewski and Brümmer leave open the natural question [1, Question 1.8(2)] whether the admissible functorial quasi-uniformity $\mathscr{C}_{b}$ on the completely regular bispaces which is spanned by the upper quasi-uniformity of the real line is always transitive for a strongly zero-dimensional bispace. It is the aim of the present note to show that their question has an affirmative answer and to discuss various aspects of this result.

In the following, expressions of the form $] a, b[$ and $[a, b[$ denote intervals of the real line. By $\mathbb{N}$ (respectively $\mathbb{Z}, \mathbb{R}$ ) we denote the set of positive integers (respectively integers, reals). Furthermore for a given real number $r$ we use the symbol $\lfloor r\rfloor$ (respectively $[r\rceil$ ) to denote the greatest integer $\leq r$ (respectively the smallest integer $\geq r$ ). For any space $X$, any map $f: X \rightarrow \mathbb{R}$ and any positive real number $\varepsilon$ we set $U_{\langle\varepsilon, f\rangle}=\{(x, y) \in$ $X \times X: f(x)-f(y)<\varepsilon\}$.

The following notation and terminology from $[1,6]$ will be used throughout. We denote the two topologies of a bispace $X$ by $\mathscr{Q}_{1} X$ and $\mathscr{Q}_{2} X$. A bispace $X$ is called zero-dimensional [13] if each member of $\mathscr{G}_{i} X$ is a union of members of $\mathscr{O}_{i} X$ whose complements belong to $\mathscr{O}_{k} X \quad(i \neq k ; i, k=1,2)$ and it is called strongly zero-dimensional [1] if its bispace Stone-Čech compactification $\beta_{b} X$ is zero-dimensional. By $T_{b}$ we denote the forgetful functor from the category Quu of quasi-uniform spaces and quasi-uniformly continuous maps to the category $\mathbf{C r}$ 2Top of completely regular bispaces and bicontinuous maps. Given a quasi-uniform space $X$ with quasi-uniformity $\mathscr{U}$ we suppose that the first topology of $T_{b} X$ is equal to $\mathscr{T}(\mathscr{U})$ and the second topology of $T_{b} X$ is equal to $\mathscr{T}\left(\mathscr{U}^{-1}\right)$.

We finish this introduction with a remark that sheds some light on the general nature of the questions discussed in this note.

During our investigations it will become clear that most problems studied in this paper are special cases of the following general problem: given a quasi-uniform space $X$ with the property that the finest totally bounded quasi-uniformity coarser than the quasi-uniformity of $X$ is transitive, determine sufficient conditions that $X$ is a transitive quasi-uniform space. The following simple (countable) example shows that we cannot expect such an implication to hold without any further assumptions.

EXAMPLe 1 [11]. Let $X$ be the subset $\{k+s / k: k \in \mathbb{N}$ and $s \in\{0, \ldots$, $k-1\}\}$ of the set of rational numbers. Define a quasi-pseudo-metric [6, p. 3] $d$ on $X$ as follows:

$$
d(x, y)= \begin{cases}0 & \text { if } x \leq y, \\ x-y & \text { if } x>y .\end{cases}
$$

Denote by $\mathscr{U}$ the quasi-uniformity on $X$ generated by the base $\left\{U_{n}: n \in\right.$ 
$\mathbb{N}\}$ where $U_{n}=\{(x, y) \in X \times X: d(x, y)<1 / n\}$ whenever $n \in \mathbb{N}$. It is known (and easy to see) that the space $(X, \mathscr{U})$ is not transitive (see [11, Example 1.2]). However since the topology $\mathscr{T}(\mathscr{U})$ is hereditarily compact, the bispace given by the topologies $\mathscr{T}(\mathscr{U})$ and $\mathscr{T}\left(\mathscr{U}^{-1}\right)$ on $X$ admits a unique compatible totally bounded quasi-uniformity, namely the (transitive) Pervin quasi-uniformity of $\mathscr{T}(\mathscr{U})$ (see, for example, [8]). Hence the finest totally bounded quasi-uniformity on the set $X$ coarser than $\mathscr{U}$ is transitive [6, Remark 1.37], although $\mathscr{U}$ does not have a transitive base.

\section{The $T_{b}$-section $\mathscr{C}_{b}$}

In this note we shall make use of the following explicit description of the $T_{b}$-section $\mathscr{C}_{b}:$ Cr2Top $\rightarrow$ Quu spanned by the real line equipped with its upper quasi-uniformity $\mathscr{Q}$. (Since terminology does not seem to be standardized, we mention that by $\mathscr{Q}$ we mean the quasi-uniformity on $\mathbb{R}$ generated by the base $\left\{Q_{\varepsilon}: \varepsilon>0\right\}$, where $Q_{\varepsilon}=\{(x, y) \in \mathbb{R} \times \mathbb{R}: x-y<\varepsilon\}$ whenever $\varepsilon$ is a positive real number (see $[6$, p. 1], but compare $[2$, p. 65] and [1, Section 1]).): Let $\mathbb{R}_{b}$ be the bispace defined on $\mathbb{R}$ whose first topology is $\mathscr{T}(\mathscr{Q})$ and whose second topology is $\mathscr{T}\left(\mathscr{Q}^{-1}\right)$. For an arbitrary bispace $X \in \mathbf{C r} 2$ Top the quasi-uniformity of $\mathscr{C}_{b} X$ is the filter on $X \times X$ generated by all sets $U_{\langle\varepsilon, f\rangle}$ where $\varepsilon$ is a positive real number and $f: X \rightarrow \mathbb{R}_{b}$ is bicontinuous.

Moreover in the proof of Proposition 1 we shall need the fact that $\mathscr{E}_{b}^{*}=$ $p_{b} \mathscr{E}_{b}$ where $p_{b}$ is the totally bounded reflection in Quu and $\mathscr{C}_{b}^{*}$ is the coarsest $T_{b}$-section (see, for example, [1]). It is known that for any $X \in \mathrm{Cr}$ 2Top the quasi-uniformity of the space $\mathscr{C}_{b}^{*} X$ is the filter on $X \times X$ generated by all sets $U_{\langle\varepsilon, f\rangle}$ where $\varepsilon$ is a positive real number and $f: X \rightarrow \mathbb{I}_{b}$ is bicontinuous [3, p. 53]. Here $\mathbb{I}_{b}$ denotes the bisubspace of $\mathbb{R}_{b}$ induced on the unit interval $\mathbb{I}$ of $\mathbb{R}$.

After these preparations we are ready to answer the question of Banaschewski and Brümmer stated in the introduction.

Proposition 1. For any bispace $X$ in $\mathrm{Cr} 2 \mathrm{Top}, \mathscr{C}_{b} X$ is transitive if and only if $X$ is strongly zero-dimensional.

Proof. Let $X \in \mathbf{C r} 2$ Top be strongly zero-dimensional. Consider an arbitrary subbasic entourage $U_{\langle\varepsilon, f\rangle}$ of $\mathscr{C}_{b} X$ of the kind described above. Clearly for each $n \in \mathbb{Z}$ we have that $U_{\langle\varepsilon / 3, f\rangle} \cap\left(f^{-1}\right](n \varepsilon / 2)+(\varepsilon / 3), \infty[$ $\left.\times f^{-1}\right]-\infty, n \varepsilon / 2[)=\varnothing$. Hence $\left.f^{-1}\right](n \varepsilon / 2)+(\varepsilon / 3), \infty[$ is far from 
$\left.f^{-1}\right]-\infty, n \varepsilon / 2\left[\right.$ with respect to the induced quasi-proximity on $\mathscr{C}_{b} X$ [6, Proposition 1.28]. Since $\mathscr{C}_{b}^{*}=p_{b} \mathscr{C}_{b}$, the two quasi-uniform spaces $\mathscr{C}_{b}^{*} X$ and $\mathscr{C}_{b} X$ carry the same induced quasi-proximity [6, Remark 1.37]. In light of the transitivity of $\mathscr{C}_{b}^{*} X$ we can choose for each $n \in \mathbb{Z}$ a transitive entourage $H_{n}$ of $\mathscr{C}_{b}^{*} X$ such that $\left.Z_{n} \cap f^{-1}\right]-\infty, n \varepsilon / 2\left[=\varnothing\right.$ where we use $Z_{n}$ to denote the set $H_{n}\left(f^{-1}\right](n \varepsilon / 2)+(\varepsilon / 3), \infty[)$. Note that $\bigcap_{n \in \mathbb{Z}} Z_{n}=\varnothing$ and $\bigcup_{n \in \mathbb{Z}} Z_{n}=X$, because $\left.\bigcup_{n \in \mathbb{Z}} f^{-1}\right]-\infty, n \varepsilon / 2\left[=X\right.$ and $\left.\bigcup_{n \in \mathbb{Z}} f^{-1}\right](n \varepsilon / 2)+$ $(\varepsilon / 3), \infty\left[=X\right.$. Furthermore $Z_{n} \subseteq f^{-1}\left[n \varepsilon / 2, \infty\left[\subseteq f^{-1}\right]((n-1) \varepsilon / 2)+\right.$ $(\varepsilon / 3), \infty\left[\subseteq Z_{n-1}\right.$ whenever $n \in \mathbb{Z}$.

For each $n \in \mathbb{Z}$ the set $Z_{n}$ belongs to the first topology of $X$ and the set $X \backslash Z_{n}$ belongs to the second topology of $X$, because $H_{n}$ is a transitive entourage of $\mathscr{C}_{b}^{*} X$. Define a map $g: X \rightarrow \mathbb{R}$ by setting $g(x)=$ $\max \left\{n \in \mathbb{Z}: x \in Z_{n}\right\}$ whenever $x \in X$. From the definition of $g$, it is straightforward to check that $\left.g^{-1}\right] a, \infty\left[=Z_{\lfloor a+1\rfloor}\right.$ and $\left.g^{-1}\right]-\infty, a[=$ $X \backslash Z_{\lceil a\rceil}$ whenever $a$ is a real number. Consequently the map $g: X \rightarrow \mathbb{R}_{b}$ is bicontinuous and $U_{\langle 1, g\rangle}$ is an entourage of $\mathscr{C}_{b} X$.

Let $(x, y),(y, z) \in U_{\langle 1, g\rangle}$. Then $g(x)-g(y)<1$ and $g(y)-g(z)<1$. Since the image of $g$ consists of integers, we deduce that $g(x)-g(y) \leq 0$ and $g(y)-g(z) \leq 0$. Therefore $g(x)-g(z) \leq 0$ and $(x, z) \in U_{\langle 1, g\rangle}$. We conclude that $U_{\langle 1, g\rangle}$ is transitive.

Finally we show that $U_{\langle 1, g\rangle} \subseteq U_{\langle\varepsilon, f\rangle}$. Let $(x, y) \in U_{\langle 1, g\rangle}$. We have $g(y)=n$ for some $n \in \mathbb{Z}$. It follows that $f(y) \geq n \varepsilon / 2$, because $y \in Z_{n} \subseteq$ $\left.f^{-1}\right] n \varepsilon / 2, \infty\left[\right.$. Since $g(x)-g(y)<1$ and $g(y)=n$, we see that $x \notin Z_{n+1}$. Hence $f(x) \leq((n+1) \varepsilon / 2)+(\varepsilon / 3)$ by the definition of $Z_{n+1}$. We get that $f(x)-f(y) \leq(\varepsilon / 2)+(\varepsilon / 3)<\varepsilon$ and $(x, y) \in U_{\langle\varepsilon, f\rangle}$. Thus we have shown that $\mathscr{C}_{b} X$ is transitive.

Since $\mathscr{C}_{b}^{*}=p_{b} \mathscr{C}_{b}$ and $p_{b}$ preserves transitivity [6, Lemma 6.3], the converse follows from Proposition 1.4 of [1] (cited in the introduction).

REMARK 1. In [1] it is observed that a positive answer to [1, Question 1.8(2)] also settles [1, Question 1.8(3)] in the affirmative: clearly Proposition 1 implies that the finest $T_{b}$-section (called $G$ in [1]) which puts transitive quasi-uniformities on all strongly zero-dimensional bispaces in Cr2Top is finer than $\mathscr{C}_{b}$. Moreover $G$ is distinct from $\mathscr{C}_{b}$ by [1, Proposition 1.7].

Note that, implicitly, in the proof of Proposition 1 we have used the following characterization of strong zero-dimensionality (in Cr2Top).

LEMmA 1. A bispace $X \in \mathbf{C r}$ 2Top is strongly zero-dimensional if and only if for each bicontinuous map $f: X \rightarrow \mathbb{I}_{b}$ there exists a set $U \in \mathscr{O}_{1} X$ such that 
$X \backslash U \in \mathscr{O}_{2} X, f^{-1}\{0\} \subseteq X \backslash U$ and $f^{-1}\{1\} \subseteq U$.

Proof. Let $X \in \mathbf{C r} 2$ Top be strongly zero-dimensional and let $f: X \rightarrow \mathbb{I}_{b}$ be bicontinuous. Since $\mathscr{E}_{b}^{*} X$ is a transitive quasi-uniform space [1, Proposition 1.4], it possesses a transitive entourage $T$ such that $T \subseteq U_{\langle 1, f\rangle}$. Set $U=T\left(f^{-1}\{1\}\right)$. Then $U \in \mathscr{O}_{1} X, X \backslash U \in \mathscr{O}_{2} X, f^{-1}\{0\} \subseteq X \backslash U$ and $f^{-1}\{1\} \subseteq U$.

In order to prove the converse let $X$ be a completely regular bispace with the stated property for bicontinuous maps from $X$ to $\mathbb{I}_{b}$ and let $A$ and $B$ be subsets of $X$ such that $A$ is far from $B$ with respect to the induced quasi-proximity on the quasi-uniform space $\mathscr{C}_{b}^{*} X$. By [6, Lemma 1.57] there exists a bicontinuous map $f: X \rightarrow \mathbb{I}_{b}$ such that $f(B)=0$ and $f(A)=1$. Moreover, by our assumption on $X$ there is a set $U \in \mathscr{O}_{1} X$ such that $X \backslash U \in$ $\mathscr{Q}_{2} X, B \subseteq X \backslash U$ and $A \subseteq U$. Define a map $g: X \rightarrow \mathbb{I}_{b}$ by setting $g(x)=1$ if $x \in U$ and $g(x)=0$ if $x \in X \backslash U$. Clearly $g: X \rightarrow \mathbb{I}_{b}$ is bicontinuous, $U_{\langle 1, g\rangle}$ is transitive and $U_{\langle 1, g\rangle} \subseteq(X \times X) \backslash(A \times B)$. By [6, Theorem 1.33] we conclude that the quasi-uniform space $\mathscr{C}_{b}^{*} X$ is transitive. Hence $X$ is strongly zero-dimensional according to [1, Proposition 1.4].

Lemma 1 should be compared with the corresponding topological result [4, Theorem 6.2.4] and analogous bitopological characterizations due to A. A. Fora [7, Theorem 3.12]. (In order to compare our result with Fora's results it may be useful to recall the following fact [12, Proposition 2.8]: if $X$ is a bispace, $f, g: X \rightarrow \mathbb{I}_{b}$ are bicontinuous maps and $f^{-1}\{0\} \cap g^{-1}\{1\}=\varnothing$, then the map $h: X \rightarrow \mathbb{I}_{b}$ defined by $h(x)=f(x) /(1-g(x)+f(x))$ whenever $x \in X$ is bicontinuous and has the properties that $f^{-1}\{0\}=h^{-1}\{0\}$ and $g^{-1}\{1\}=h^{-1}\{1\}$.)

REMARK 2. The semi-continuous quasi-uniformity of a topological space $X$ is the filter on $X \times X$ generated by all sets $U_{\langle\varepsilon, f\rangle}$ where $\varepsilon$ is a positive real number and $f: X \rightarrow(\mathbb{R}, \mathscr{T}(\mathscr{Q}))$ is continuous [6, p. 32]. Often a different characterization of the semi-continuous quasi-uniformity is more convenient. In order to formulate this characterization we recall that an open spectrum $\mathscr{A}$ in a topological space $X$ is a sequence $\left\{A_{n} ; n \in \mathbb{Z}\right\}$ of open sets of $X$ such that $\bigcap_{n \in \mathbb{Z}} A_{n}=\varnothing, \cup_{n \in \mathbb{Z}} A_{n}=X$ and $A_{n} \subseteq A_{n+1}$ whenever $n \in \mathbb{Z}\left[6\right.$, p. 33]. Furthermore, let us say that $U_{\mathscr{A}}=\bigcup_{n \in \mathbb{Z}}\left[\left(A_{n} \backslash A_{n-1}\right) \times A_{n}\right]$ is the neighbournet associated with the open spectrum $\mathscr{A}$ in $X$. In [5] $P$. Fletcher and W. F. Lindgren show that the semi-continuous quasi-uniformity of a topological space $X$ is the compatible quasi-uniformity on $X$ that is generated by all neighbournets associated with open spectra in $X$ (compare also [6, Theorem 2.12]). In the remaining paragraphs of this section we wish 
to show that the method employed in the proof of Proposition 1 yields a corresponding result for bispaces, which extends the result of Fletcher and Lindgren. In order to simplify the formulation of Proposition 2 we introduce the following auxiliary concept.

Definition 1. A bispectrum $\mathscr{A}$ in a bispace $X$ is a sequence $\mathscr{A}=$ $\left\{A_{n} ; n \in \mathbb{Z}\right\}$ of subsets of $X$ such that both $\mathscr{A}$ is an $\mathscr{O}_{1} X$-open spectrum in $X$ and $\mathscr{A}^{C}=\left\{X \backslash A_{-n} ; n \in \mathbb{Z}\right\}$ is an $\mathscr{O}_{2} X$-open spectrum in $X$.

Proposition 2. A bispace $Y \in \mathbf{C r} 2$ Top is strongly zero-dimensional if and only if the quasi-uniformity of the space $\mathscr{C}_{b} Y$ is generated by all transitive relations of the form $U_{\mathscr{A}}$ on $Y$ where $\mathscr{A}$ is running through all bispectra in $\boldsymbol{Y}$.

Proof. Note that the sequence $\mathscr{Z}=\left\{Z_{-n} ; n \in \mathbb{Z}\right\}$ considered in the proof of Proposition 1 is a bispectrum in $X$. Moreover $\mathscr{Z}$ and its associated map $g$ have the property that $U_{\langle 1, g\rangle}=U_{\mathscr{X}}$, since clearly $U_{\langle 1, g\rangle}(x)=Z_{g(x)}$ whenever $x \in X$. The assertion is now an immediate consequence of the proof of Proposition 1.

REMARK 3. For a given topological space $X$ let $Q_{1} X$ be the completely regular bispace defined on the ground set of $X$ whose first topology is that of $X$ and whose second topology has the closed sets of $X$ as a base for open sets. It is known that $Q_{1} X$ is strongly zero-dimensional [1, Proposition 1.5]. By Proposition 2 the quasi-uniformity of $\mathscr{C}_{b} Q_{1} X$ is generated by all neighbournets $U_{\mathscr{A}}$ where $\mathscr{A}$ is running through all bispectra in $Q_{1} X$. In order to see that, essentially, this last statement is equivalent to the above-mentioned result of Fletcher and Lindgren, observe now that the quasi-uniformity of $\mathscr{C}_{b} Q_{1} X$ is equal to the semi-continuous quasi-uniformity of $X$ (see [14, Proposition 4.1]) and that the set of bispectra in $Q_{1} X$ consists of the set of open spectra in $X$.

\section{The fine $T_{b}$-section}

Since the finest compatible quasi-uniformity on a strongly zero-dimensional bispace $X \in \mathbf{C r}$ Top need not be transitive [1, Proposition 1.6], it seems natural to consider the following question.

Question. Which strongly zero-dimensional bispaces in Cr2Top have the property that their fine quasi-uniformity is transitive?

Of course such a general question can hardly have a simple answer. Note that the seemingly extremely difficult problem to decide whether a given 
topological space $X$ is transitive [6, Chapter 6] is part of this problem, because the finest (transitive) compatible quasi-uniformity on the strongly zerodimensional bispace $\mathscr{Q}_{1} X$ is equal to the fine (transitive) quasi-uniformity of $X$ (compare Remark 3 and [14]). However, as we are going to show next, in two important special classes of strongly zero-dimensional bispaces the question can be dealt with easily.

Recall that a topology is called pseudo- $\aleph_{1}$-compact if each locally finite family of non-empty open sets is countable.

Proposition 3. The finest compatible quasi-uniformity on a strongly zerodimensional bispace $X \in \mathbf{C r} 2 \mathrm{Top}$ is transitive if the topology $\mathscr{O}_{1} X \vee \mathscr{O}_{2} X$ is pseudo- $\aleph_{1}$-compact.

Proof. Let $X \in \mathbf{C r} 2 \mathrm{Top}$ be a strongly zero-dimensional bispace such that $\mathscr{O}_{1} X \vee \mathscr{O}_{2} X$ is pseudo- $\aleph_{1}$-compact. Consider an arbitrary entourage $V$ belonging to the finest compatible quasi-uniformity on $X$. Since the quasiproximity induced by this quasi-uniformity is equal to the quasi-proximity induced on the transitive [1, Proposition 1.4] space $\mathscr{C}_{b}^{*} X$, for each $x \in X$ there are $G_{x} \in \mathscr{O}_{1} X$ and $G_{x}^{\prime} \in \mathscr{O}_{2} X$ such that $X \backslash G_{x} \in \mathscr{O}_{2} X, X \backslash G_{x}^{\prime} \in$ $\mathscr{Q}_{1} X, V(x) \subseteq G_{x} \subseteq V^{2}(x)$ and $V^{-1}(x) \subseteq G_{x}^{\prime} \subseteq V^{-2}(x)$ (see proof of Proposition 1). By our assumption on the topology $\mathscr{O}_{1} X \vee \mathscr{O}_{2} X$ the normal cover $\left\{\left(V \cap V^{-1}\right)(x): x \in X\right\}$ of the topological space $\left(X, \mathscr{O}_{1} X \vee \mathscr{O}_{2} X\right)$ has a countable subcover $\left\{\left(V \cap V^{-1}\right)\left(x_{i}\right): i \in \mathbb{N}\right\}$. For each $k \in \mathbb{N}$ set $T_{k}=\left(\left[\left(X \backslash G_{x_{k}}\right) \times X\right] \cup\left[X \times G_{x_{k}}\right]\right) \cap\left(\left[G_{x_{k}}^{\prime} \times X\right] \cup\left[X \times\left(X \backslash G_{x_{k}}^{\prime}\right)\right]\right)$. Let $S_{i}=$ $\bigcap\left\{T_{k}: k \in \mathbb{N}\right.$ and $\left.k \leq i\right\}$ whenever $i \in \mathbb{N}$. Note that each relation $S_{i}$ is transitive. For each $y \in X$ let $i(y)$ be the minimal $i \in \mathbb{N}$ such that $y \in\left(V \cap V^{-1}\right)\left(x_{i}\right)$. One checks that $S=\bigcup_{y \in X}\left[S_{i(y)}^{-1}(y) \times S_{i(y)}(y)\right]$ is transitive and belongs to the finest compatible quasi-uniformity on $X$. Furthermore $S_{i(y)}^{-1}(y) \times S_{i(y)}(y) \subseteq G_{x_{i(y)}}^{\prime} \times G_{x_{i(y)}} \subseteq V^{-2}\left(x_{i(y)}\right) \times V^{2}\left(x_{i(y)}\right) \subseteq V^{4}$ whenever $y \in X$. We conclude that the finest quasi-uniformity which the bispace $X$ admits is transitive.

COROLlary 1. The finest compatible quasi-uniformity on a countable bispace in Cr2Top is transitive.

Proof. Let $X \in \mathbf{C r}$ 2Top be countable and let $f: X \rightarrow \mathbb{I}_{b}$ be bicontinuous. Since $X$ is countable, there exists $x \in \mathbb{I}_{b} \backslash(f(X) \cup\{0,1\})$. Set $\left.\left.U=f^{-1}\right] x, 1\right]$. Then $U \in \mathscr{O}_{1} X, X \backslash U=f^{-1}\left[0, x\left[\in \mathscr{O}_{2} X, f^{-1}\{0\} \subseteq X \backslash U\right.\right.$ and $f^{-1}\{1\} \subseteq U$. The result follows from Lemma 1 and Proposition 3 . 
In the final paragraphs of this note we shall use the following notation. Given a quasi-pseudo-metric space $(X, d)$, for each $x \in X$ and each $n \in \mathbb{N}$ we denote the set $\left\{y \in X: d(x, y)<2^{-n}\right\}$ by $B_{d}(n, x)$. Furthermore for any subset $A$ of $X$ and any $n \in \mathbb{N}$ we set $B_{d}(n, A)=\bigcup\left\{B_{d}(n, a): a \in A\right\}$.

Proposition 4. The finest compatible quasi-uniformity on a non-archimedeanly quasi-pseudo-metrizable bispace is transitive. In particular each nonarchimedeanly quasi-pseudo-metrizable bispace is strongly zero-dimensional.

Proof. Let $X$ be a non-archimedeanly quasi-pseudo-metrizable bispace and let $V$ be an entourage belonging to the finest compatible quasi-uniformity on $X$. Choose a non-archimedean quasi-pseudo-metric $d$ on $X$ such that $\mathscr{T}(d)=\mathscr{O}_{1} X$ and $\mathscr{T}\left(d^{-1}\right)=\mathscr{O}_{2} X$. For each $x \in X$ there exists an $n_{x} \in \mathbb{N}$ such that $B_{d}\left(n_{x}, x\right) \subseteq V(x)$ and $B_{d^{-1}}\left(n_{x}, x\right) \subseteq V^{-1}(x)$. Set $T=\bigcup_{x \in X}\left[B_{d^{-1}}\left(n_{x}, x\right) \times B_{d}\left(n_{x}, x\right)\right]$. Then $T$ is transitive, $T$ is a subset of $V^{2}$ and $T$ belongs to the finest compatible quasi-uniformity on $X$. Hence we have shown that the finest compatible quasi-uniformity on $X$ is transitive. The second assertion is a consequence of [6, Lemma 6.3] and [1, Proposition 1.4], because the quasi-uniformity of $\mathscr{C}_{b}^{*} X$ is the finest totally bounded quasi-uniformity that $X$ admits.

COROLlary 2. Let $\mathscr{V}$ be a quasi-uniformity with a countable base on a set $X$ and let $\mathscr{S}$ be a transitive quasi-uniformity on $X$ such that $\mathscr{S} \subseteq \mathscr{V}$, $\mathscr{T}(\mathscr{V})=\mathscr{T}(\mathscr{S})$ and $\mathscr{T}\left(\mathscr{V}^{-1}\right)=\mathscr{T}\left(\mathscr{S}^{-1}\right)$. Then the bispace given by the two topologies $\mathscr{T}(\mathscr{V})$ and $\mathscr{T}\left(\mathscr{V}^{-1}\right)$ on $X$ is non-archimedeanly quasipseudo-metrizable. In particular its fine quasi-uniformity is transitive.

Proof. Let $\left\{V_{n}: n \in \mathbb{N}\right\}$ be a countable base of the quasi-uniformity $\mathscr{V}$. One checks that $\left\{\bigcup_{k=1}^{\infty} V_{n}^{k}: n \in \mathbb{N}\right\}$ is a countable transitive base for a quasiuniformity $\mathscr{K}$ on $X$ such that $\mathscr{T}(\mathscr{K})=\mathscr{T}(\mathscr{V})$ and $\mathscr{T}\left(\mathscr{K}^{-1}\right)=\mathscr{T}\left(\mathscr{V}^{-1}\right)$ (compare [9, proof of Proposition]). Hence the bispace under consideration is non-archimedeanly quasi-pseudo-metrizable (see, for example, the proof of Proposition 5 below).

Our last result generalizes the well-known fact that each strongly zerodimensional metrizable space admits a non-archimedean metric (see, for example, [6, Theorem 6.8]). The proof of our result suggests that the difficulties which we face when studying the problem mentioned in the beginning of this section are caused mainly by the fact that in the bitopological setting there does not exist a satisfactory analogue of the topological concept of paracompactness (compare with the proof of [6, Theorem 6.4]). 
Proposition 5. Let $X$ be a quasi-pseudo-metrizable bispace that is strongly zero-dimensional. If both $\mathscr{O}_{1} X$ and $\mathscr{O}_{2} X$ have a $\sigma$-point-finite base, then $X$ is non-archimedeanly quasi-pseudo-metrizable.

Proof. Let $d$ be a quasi-pseudo-metric on $X$ such that $\mathscr{O}_{1} X=\mathscr{T}(d)$ and $\mathscr{O}_{2} X=\mathscr{T}\left(d^{-1}\right)$. To begin, note that for each $r \in \mathbb{N},\{(x, y) \in X \times X$ : $\left.d(x, y)<2^{-r}\right\}$ belongs to the finest compatible quasi-uniformity on the bispace $X$. Therefore for any subset $A$ of $X$ and any $r \in \mathbb{N}$ the set $A$ is far from $X \backslash B_{d}(r, A)$ with respect to the induced quasi-proximity on $\mathscr{C}_{b}^{*} X$.

Let $\mathscr{B}=\bigcup_{n \in \mathrm{N}} \mathscr{B}_{n}$ be a base for $\mathscr{O}_{1} X$ such that $\mathscr{B}_{n}$ is point-finite for each $n \in \mathbb{N}$. Fix $n, m \in \mathbb{N}$ and consider an arbitrary $B \in \mathscr{B}_{n}$. Set $B_{m}=\{x \in X$ : $\left.B_{d}(m, x) \subseteq B\right\}$. Since $X$ is strongly zero-dimensional, it follows (compare with the proof of Proposition 1) that we can choose a set $G(m, B) \in \mathscr{O}_{1} X$ such that $X \backslash G(m, B) \in \mathscr{O}_{2} X$ and $B_{m} \subseteq G(m, B) \subseteq B_{d}\left(m+1, B_{m}\right)$. Set $\mathscr{H}_{m, n}=\left\{G(m, B): B \in \mathscr{B}_{n}\right\}$. We note that for each $n \in \mathbb{N}$ and each $B \in \mathscr{B}_{n}$ we have that $\bigcup_{m \in \mathbb{N}} G(m, B)=B$. Hence, obviously, $\bigcup_{m, n \in \mathbb{N}} \mathscr{H}_{m, n}$ is a $\sigma$-point-finite base for the topology $\mathscr{O}_{1} X$.

Next we show that each collection $\mathscr{H}_{m, n}$ is locally finite with respect to the topology $\mathscr{O}_{2} X$. Fix $m, n \in \mathbb{N}$ and $x \in X$. Assume that $G(m, B) \cap$ $B_{d^{-1}}(m+1, x) \neq \varnothing$ where $B \in \mathscr{B}_{n}$. Then $x \in B_{d}\left(m, B_{m}\right) \subseteq B$. Since the collection $\mathscr{B}_{n}$ is point-finite, it is clear that $\mathscr{H}_{m, n}$ is locally finite with respect to the topology $\mathscr{O}_{2} X$.

For each $x \in X$ and $s \in \mathbb{N}$ set $T_{s}(x)=\bigcap\left\{H: x \in H \in \mathscr{H}_{m, n}\right.$ where $m, n \in \mathbb{N}$ and $m, n \leq s\}$. (We use the convention that $\cap \varnothing=X$.) Furthermore set $T_{s}=\bigcup_{x \in X}\left[\{x\} \times T_{s}(x)\right]$ whenever $s \in \mathbb{N}$. Then $\left(T_{s}\right)_{s \in \mathbb{N}}$ is a decreasing sequence of transitive binary relations on $X$ such that $\left\{T_{s}(x)\right.$ : $s \in \mathbb{N}\}$ is an $\mathscr{O}_{1} X$-open neighbourhood base at $x$ whenever $x \in X$. Moreover, $T_{s}^{-1}(x)=X \backslash \bigcup\left\{T_{s}(y): y \in X \backslash T_{s}^{-1}(x)\right\}$ is $\mathscr{O}_{2} X$-open for each $x \in X$ and $s \in \mathbb{N}$, since each collection $\mathscr{Z}_{s}=\left\{T_{s}(y): y \in X\right\}$ is $\mathscr{O}_{2} X$-closed and each point of $X$ has an $\mathscr{O}_{2} X$-open neighbourhood hitting only finitely many different elements of $\mathscr{Z}_{s}$.

Similarly we can obtain a decreasing sequence $\left(S_{s}\right)_{s \in \mathbb{N}}$ of transitive binary relations on $X$ such that $\left\{S_{s}^{-1}(x): s \in \mathbb{N}\right\}$ is an $\mathscr{O}_{2} X$-open neighbourhood base at $x$ whenever $x \in X$ and such that $S_{s}(x)$ is $\mathscr{O}_{1} X$-open whenever $x \in X$ and $s \in \mathbb{N}$. Set $P_{s}=T_{s} \cap S_{s}$ for each $s \in \mathbb{N}$. Furthermore let $P_{0}=X \times X$. Define a compatible non-archimedean quasi-pseudo-metric $p$ on $X$ as follows. For each $x, y \in X$ set

$$
p(x, y)= \begin{cases}0 & \text { if }(x, y) \in \bigcap\left\{P_{n}: n \in \mathbb{N}\right\}, \\ 2^{-(n-1)} & \text { if }(x, y) \in P_{n-1} \backslash P_{n} \text { for some } n \in \mathbb{N} .\end{cases}
$$

We have shown that $X$ is non-archimedeanly quasi-pseudo-metrizable. 
It would be interesting to know under which conditions in Proposition 5 the assumption that both topologies $\mathscr{\sigma}_{1} X$ and $\mathscr{\sigma}_{2} X$ have a $\sigma$-point-finite base can be weakened to the condition that both topologies have a $\sigma$-interiorpreserving base.

In light of Proposition 5 also the following natural questions seem to be of interest.

Questions. Let $X \in \mathbf{C r} 2$ Top be strongly zero-dimensional such that both $\mathscr{O}_{1} X$ and $\mathscr{O}_{2} X$ have a $\sigma$-point-finite base. When is the finest compatible quasi-uniformity on $X$ transitive?

Is each topological space with a $\sigma$-point-finite base transitive (compare with [10, Corollary 1.8(b) and Remark 1.9])?

\section{Acknowledgement}

The author would like to thank Professor G. C. L. Brümmer for many valuable comments on our first draft. The present paper incorporates a number of his suggestions.

\section{References}

[1] B. Banaschewski and G. C. L. Brümmer, 'Strongly zero-dimensionality of biframes and bispaces', Quaestiones Math. 13 (1990), 273-290.

[2] G. C. L. Brümmer, 'On certain factorizations of functors into the category of quasiuniform spaces', Quaestiones Math. 2 (1977), 59-84.

[3] G. C. L. Brümmer, 'On the non-unique extension of topological to bitopological properties', Categorical Aspects of Topology and Analysis (Proc. Conf., Ottawa, 1980), Lecture Notes in Math. (Springer) 915 (1982), 50-67.

[4] R. Engelking, General Topology, Heldermann, Berlin, 1989.

[5] P. Fletcher and W. F. Lindgren, 'Quasi-uniformities with a transitive base', Pacific J. Math. 43 (1972), 619-631.

[6] P. Fletcher and W. F. Lindgren, Quasi-uniform Spaces, Lecture Notes Pure Appl. Math. 77, Marcel Dekker, New York, 1982.

[7] A. A. Fora, 'Strongly zero-dimensional bitopological spaces', J. Univ. Kuwait (Sci.) 11 (1984), 181-190.

[8] H. P. A. Künzi, 'Topological spaces with a unique compatible quasi-proximity', Arch. Math. 43 (1984), 559-561.

[9] H. P. A. Künzi, 'Topological spaces with a unique compatible quasi-uniformity', Canad. Math. Bull. 29 (1986), 40-43.

[10] H. P. A. Künzi, 'Some remarks on quasi-uniform spaces', Glasgow Math. J. 31 (1989), 309-320.

[11] H. P. A. Künzi, 'Functorial admissible quasi-uniformities on topological spaces', Top. Appl. 43 (1992), 27-36.

[12] E. P. Lane, 'Bitopological spaces and quasi-uniform spaces', Proc. London Math. Soc. 17 (1967), 241-256. 
[13] I. L. Reilly, 'Zero dimensional bitopological spaces', Nederl. Akad. Wetensch., Proc. Ser. A 76 = Indag. Math. 35 (1973), 127-131.

[14] S. Salbany, 'Quasi-uniformities and quasi-pseudometrics', Math. Colloq. Univ. Cape Town $6(1970-71), 88-102$.

\section{University of Berne}

Sidlerstrasse 5

3012 Berne

Switzerland 